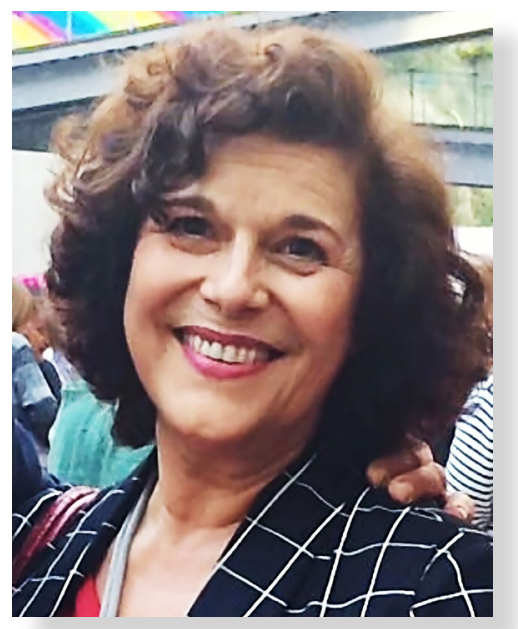

\section{¿Cómo llegan a ser profesores los estudiantes de pedagogía en educación secundaria en Chile? La práctica y la teoría en la formación del conocimiento profesional docente}

\section{How do secondary education pedagogy students become teachers in Chile? Practice and theory in the formation of professional teacher knowledge}

Recibido: 16-04-17

Margot Recabarren Herrera'

\section{Resumen}

Por la importancia del rol del profesor en toda sociedad, la formación inicial docente es un desafío, y se asume como un compromiso de calidad con las futuras generaciones. En este artículo se da cuenta de parte de una investigación llevada a cabo en el programa de Pedagogía en Educación Media (Secundaria), de formación consecutiva, que abarca la construcción del conocimiento profesional docente, a través de los procesos de práctica inicial y profesional, desde las experiencias de sus participantes, tanto alumnos como supenvisores, y aborda el impacto de las experiencias previas y la relación teoría - práctica en la conformación de este conocimiento. Es una investigación cualitativa, que se posiciona desde el paradigma interpretativo, utiliza una metodología de estudio de caso, con recogida de datos desde la entrevista y el relato (bitácoras). Para la fase de análisis de los datos y la construcción de categorías se trabaja desde la teoría fundamentada. Los hallazgos relativos a parte de la investigación que se reseña en este artículo, dan cuenta de un proceso complejo, que revaloriza las experiencias previas, la importancia del modelado docente, la relación teoría-práctica y el valor del acompañamiento cercano. El enfoque realista (Korthagen, 2010), el monitoreo cercano y la reflexión aparecen como claves para mejorar los procesos.

\title{
Abstract
}

Initial teachertraining is a challenge thatevery society assumes as a commitment to quality with future generations. This article reports on the part of a research camied out in the Pedagogy Program in Secondary Education (Rittershaussen, 2015), which includes the construction of professional teacher knowledge, through the processes of initial and professional practice, from the experiences of Its participants, both students, and supervisors. Specifically, the impact of previous experiences and the relation between practical and theory in the construction of this knowledge, as experienced by students, are addressed. This research is developed from a qualitative perspective, and its approach is based on the interpretative paradigm, uses a case study methodology and focuses mainly on the collection of data through interviews and reports (logs). For the analysis phase of the data and the construction of categories, the design of the grounded theory was followed. The findings related to part of the research outlined in this article show a complex process that reassesses previous experiences, the importance of teacher modeling, the theory-practice relationship and the value of close accompaniment. Other results illuminate the critical role of teaching planning, pupils' difficulties in "crossing borders," and the relationship between school - teacher guide - students in practice - supervisor teacher university. Realistic approach, close monitoring, and reflection appear as keys to improve processes.

\section{Palabras clave}

formación docente; proceso práctica profesional; experiencias previas; relación teoría-práctica

\section{Keywords}

teacher training; professional practice; previous experiences; theory-practice relationship

*Margot Recabarren Herrera: Es profesora de Historia y Geografía, de la PUCV, 1981, Magister en Gestión y Planificación Educacional UDP, 2006 y Magister en Ciencia Política Integrada, ACANAV, UMACH, 2006. Actualmente es candidata a grado de doctor en Planificación e Innovación Educativa, de la Universidad de Alcalá, en proceso de defensa de tesis. Se desempeña como Directora de Pedagogía Educación Media, de la Universidad Andrés Bello. Formadora de docentes desde el año 1992. Para contactar a la autora: mrecabarren@unab.cl 


\section{Introducción}

Ser profesor para mí significa ser alguien que asume el desafío de educar no solo en lo que tiene que ver con conocimientos, sino que también en lo que tiene que ver con la vida. Si logramos que nuestros estudiantes aprendan de nuestra disciplina y que además se conviertan en buenas personas, podemos decir que hemos hecho un buen trabajo.

(Gonzalo)

Los profesores importan..., y mucho. Pero ¿cómo se llega a ser profesor? ¿Qué conocimientos forman parte de ese "ser profesor"? ¿Cómo se adquieren esos conocimientos? Como se forma un docente no es un tema menor para cualquier país.

Hay mucho en juego en ello, como para no atender lo que involucra formar un profesor, especialmente en un contexto, como el chileno, en que las demandas de calidad, las reformas a la educación, la generación de estándares de egreso, la evaluación docente, la carrera docente, son todas temáticas de actual interés.

En la investigación realizada por Jesús Manso (2012) acerca de "La formación inicial del profesorado de Educación Secundaria", se respalda sin lugar a dudas, la creciente importancia de la figura del profesorado, y según el informe de la Comisión al Consejo y Parlamento Europeo, los profesores,

..., son los mediadores entre un mundo que cambia rápidamente y los alumnos que están a punto de entrar en él. La profesión docente es cada vez más compleja. Las exigencias a los profesores van en aumento. Los entornos en los que trabajan plantean cada vez más retos.

(Comisión de las Comunidades Europeas, 2007, p.2)

En este contexto, resulta relevante investigar cómo se forma un profesor de educación secundaria.

\section{Algunos factores que definen la formación del saber pedagógico}

De acuerdo a Beatrice Ávalos y Carmen Sotomayor (2011, p. 58), la "forma como los profesionales definen y asumen las tareas que les son propias y al modo como entienden sus relaciones con otras personas que cumplen las mismas tareas" son definitorias en la formación, no solo de la identidad, sino del tipo de saber que los docentes deben manejar, y que se pide de ellos.

La formación inicial docente y el desarrollo profesional de los profesores son estudiados y presentados en la literatura especializada, de muchas maneras diferentes. Pero siempre en el eje central de los diferentes estudios, estáelllegaralacomprensión decómosevaconformando tanto el inicio como el desarrollo profesional del profesor (Avalos, 2011), qué se conjuga para conformar ese aprendizaje y cómo se mantiene, se desarrolla, se construye y de-construye en el transcurso de su vida profesional.

¿Existen aprendizajes relevantes que una vez construidos se mantienen casi intactos a lo largo de su vida profesional?

Esta investigación se llevó a cabo con alumnos, egresados y supervisores del Programa de Formación Consecutiva de Pedagogía en Educación Media. Este programa ofrece formación consecutiva a quienes ya poseen una licenciatura de especialidad (Rittershaussen, 2015).

Con este estudio se buscó pesquisar cómo se construye el conocimiento profesional docente a través de los procesos de práctica. Para la investigación se seleccionaron participantes de las tres sedes, alumnos egresados, alumnos en curso (2016) y profesores supervisores.

Esta investigación se desarrolló desde una mirada cualitativa y su enfoque se basa en el paradigma interpretativo, utiliza una metodología de estudio de caso y centrada principalmente en la recogida de datos mediante las entrevistas y los relatos (bitácoras).

Para la fase de análisis de los datos y la construcción de categorías se siguió el diseño de la teoría fundamentada y se utilizó el NVivo-9 como software de apoyo al análisis cualitativo. 
Tabla 1

Participantes por sede - Organización de la búsqueda de información

\begin{tabular}{|c|c|c|}
\hline Participante 1 & Aspecto a pesquisar & Instrumento \\
\hline $\begin{array}{l}\text { Alumnos egresados } 2015 \\
\text { Sedes: VIN-CON-REP } \\
\text { (Santiago) } \\
3 \text { alumnos por sede } \\
\text { Total }=9+1=10\end{array}$ & $\begin{array}{l}\text { Experiencias significativas etapa } \\
\text { escolar. } \\
\text { Experiencia práctica inicial } \\
\text { Experiencia práctica profesoral } \\
\text { Relación teoría-práctica }\end{array}$ & Entrevista \\
\hline Participante 2 & Aspecto a pesquisar & Instrumento \\
\hline $\begin{array}{l}\text { Alumno en curso } 2016 \\
\text { Sedes: } \\
\text { Viña del Mar, } \\
\text { Concepción, Santiago } \\
3 \text { alumnos por sede } \\
\text { Total }=9+3=12\end{array}$ & $\begin{array}{l}\text { Experiencias significativas etapa } \\
\text { escolar. } \\
\text { Experiencia práctica inicial } \\
\text { Experiencia práctica profesoral } \\
\text { Relación teoría-práctica }\end{array}$ & $\begin{array}{l}\text { Entrevista individual o grupal. } \\
\text { Se entrevistará dos veces a los mismos } \\
\text { sujetos: } \\
\text { En el proceso de Práctica Inicial } \\
\text { junio-julio } \\
\text { En el Proceso de Práctica Profesional } \\
\text { diciembre-enero } 2017 \\
\text { (entrevista individual o grupal) } \\
\text { Revisión de bitácoras }\end{array}$ \\
\hline Participante 2 & Aspecto a pesquisar & Instrumento \\
\hline $\begin{array}{l}\text { Profesor Supervisor } \\
4 \text { supervisores } \\
\text { por sede }=12 \\
\text { Sedes: VIN-CON-REP }\end{array}$ & $\begin{array}{l}\text { Experiencias como supervisor } \\
\text { Experiencia práctica profesional } \\
\text { Proceso de llegar a ser profesor, } \\
\text { dificultades que ve en el alumno } \\
\text { Relación teoría-práctica }\end{array}$ & Entrevista \\
\hline
\end{tabular}

El levantamiento de información se realizó a través de entrevistas. Se hizo trascripción y un primer análisis que permitió levantar las primeras categorías, y emergieron algunas como "experiencias previas", "encuentro teoríapráctica", "la realidad de aula", etc. Para el trabajo con la información se realizó un levantamiento y análisis de categorías y subcategorías, según las orientaciones de Flick (2004), abierta, axial y selectiva. Se trabajó con levantamiento de categorías por grupo de informantes (egresados, alumnos en curso, supervisores, alumnos en curso pero con experiencia pedagógica), y después se cruzaron las informaciones de los diferentes hablantes a partir de un eje de análisis. Una vez realizado el cruce, se volvió a reunir la información desde los ejes, pero esta vez por sede de origen y especialidad. Finalmente, se volvieron a juntar y se seleccionaron los textos representativos de cada eje. En la triangulación final se toman en consideración entonces el informante, su condición, su origen y su especialidad. En este artículo se da cuenta de parte del proceso y de algunos de los resultados obtenidos.

El profesor chileno, de acuerdo a los datos de la OCDE 2014 $(D 6,524)$, es uno de los que más horas de contacto de aula tiene al año, tiene a su vez, menos horas para preparar su propuesta pedagógica y didáctica, planificar, diseñar materiales, revisar y corregir evaluaciones, y es el que atiende a grupos más numerosos; pero, a pesar de ello, forma parte del grupo de docentes que se sienten bien preparados..., "los profesores opinan que la formación que recibieron les prepara bien para el desempeño de su profesión. Un promedio del 93\% de los profesores afirmaron

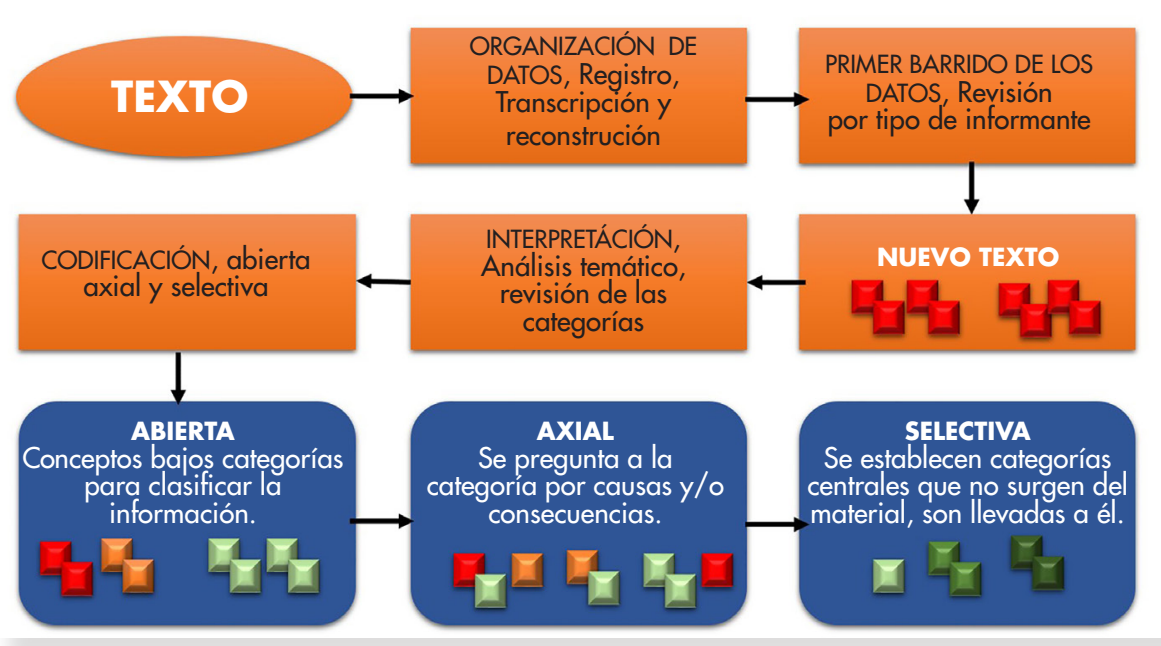

Figura 1 Secuencia Organización de la Información, basada en texto de Flick (2004). y reseña de Castiblanco y Vizcaíno (2010)

“¿Cómo llegan a ser profesores los estudiantes de pedagogía en educación secundaria en Chile? La práctica y la teoría en la formación del conocimiento profesional docente", Margot Recabarren, Cuaderno de Pedagogía Universitaria Vol. 13/ no. 26/ julio-diciembre 2016/ República Dominicana/ PUCMM/ ISSN 1814-4152 (en línea) / ISSN 1814-4144 (impresa) / p. 26-35 
sentirse bien preparados o muy bien preparados para enseñar el contenido de las asignaturas que imparten y el $89 \%$ se sienten bien preparados o muy bien preparados en la pedagogía y en la práctica de dichas asignaturas" (OCDE, 2104).

El cuestionamiento de base es cómo entonces se construye ese aprendizaje, y qué aspectos del proceso de práctica en su etapa formativa, consideran ellos que es relevante para ser profesor.

Según Imbernon (2007), partimos de la base de que:

la función docente comporta un conocimiento pedagógico específico, un compromiso ético y moral y la necesidad de corresponsabilización con otros agentes sociales; esto es así puesto que ejerce influencia sobre otros seres humanos $y$, por esta razón, no puede ni debe ser una función meramente técnica de expertos infalibles (2007, p.23).

Además, cuenta también con un componente práctico, ya que los profesores y profesoras adquieren un conjunto de conocimientos y habilidades especializadas durante un período de formación a lo largo de la vida profesional como resultado de una intervención en un contexto y una toma de decisiones aplicadas a situaciones únicas y particulares que encuentran durante el desempeño de su labor.

La función docente se desarrolla entonces en un equilibrio entre las tareas profesionales, la activación y aplicación de un conocimiento disciplinar específico (Imbernon, 2007), la realidad de aula en que se aplican, el compromiso ético de su función social y la estructura de participación social existente en ese momento y que se reconoce en la unidad educativa como contexto inmediato y con la cual está comprometido.

Pero, ¿cómo se aprende a enseñar? Para Lourdes Montero, aprender a enseñar puede ser definido como: "un proceso que implica efectivamente la adquisición de un repertorio de conocimientos, habilidades, actitudes, creencias y afectos construidos a lo largo y ancho del ejercicio profesional de cualquier profesor, en estrecha relación con los diversos contextos en los que éste tiene lugar." (Montero, 2001, p.153)

Ya Calvo \& Camargo (2015) en su investigación sobre la formación de docentes, pudieron determinar que los profesores nóveles, frente a los retos que deben enfrentar, propios de las dinámicas del contexto educativo donde están insertos, en la práctica que desarrollan, "recomponen saberes -saberes en acciónque los llevan a leer los contextos y a desarrollar las competencias -saberes de innovación- que necesitan en el ejercicio de hacerse profesionales de la docencia" (p. 2).

\section{El rol de las experiencias previas en la formación del saber pedagógico}

Según Carlos Marcelo (2006), el conocimiento y las creencias se construyen, y los profesores, así como otras personas, orientan y regulan su conducta a partir del conjunto de conocimientos y creencias que les son propios. Este aprendizaje inconsciente y estas creencias se empiezan a construir desde las experiencias vividas, mucho antes que el "futuro profesor" decida dedicarse profesionalmente a la enseñanza.

El aprendizaje en muchos casos no se produce de manera intencionada en los estudiantes, sino que es la resultante de experiencias, las más de las veces obtenidas en la vida estudiantil, que los marcan significativamente: pocos olvidan a un mal profesor.

A su vez, un buen profesor suele dejar una huella indeleble. Ya sea por el ejemplo o el contraejemplo, el futuro profesor/a ha acumulado experiencias previas (Alsina et al. 2016), que se transforman en conocimiento de vida, que suele estar a la base de las creencias sobre lo que es ser docente.

Estos conocimientos y creencias que los profesores en formación traen consigo cuando inician su formación inicial afectan de una manera directa a la interpretación y valoración que los profesores hacen de las experiencias de formación del profesorado. Esta modalidad de aprender a enseñar se produce a través de lo que se ha denominado aprendizaje por observación. Aprendizaje que en muchas ocasiones no se produce de manera intencionada, sino que se va adentrando en las estructuras cognitivas -y emocionales- de los futuros profesores de manera inconsciente, llegando a crear expectativas y creencias difíciles de remover. (Marcelo, 2006, p. 73)

Evidencias de estas formaciones previas y del impacto en el desempeño docente fueron encontradas en la investigación recientemente realizada con los alumnos del Programa de Pedagogía en Educación Media, de la Universidad Andrés Bello. Estos conocimientos y creencias que los profesores en formación traen consigo cuando inician su formación inicial afectan de una manera directa a la interpretación y valoración que los profesores hacen de las experiencias de formación del profesorado.

Esta modalidad de aprender a enseñar se produce a través de lo que se ha denominado aprendizaje por observación. Aprendizaje que en muchas ocasiones no se produce de manera intencionada, sino que se va adentrando en las estructuras de cognitivas -y emocionales- de los futuros profesores de manera inconsciente, llegando a crear expectativas y creencias difíciles de remover. (Marcelo, 2006, p. 73)

“¿Cómo llegan a ser profesores los estudiantes de pedagogía en educación secundaria en Chile? La práctica y la teoría en la formación del conocimiento profesional docente", Margot Recabarren, Cuaderno de Pedagogía Universitaria Vol. 13/ no. 26/ julio-diciembre 2016/ República Dominicana/ PUCMM/ ISSN 1814-4152 (en línea) / ISSN 1814-4144 (impresa) / p. 26-35 


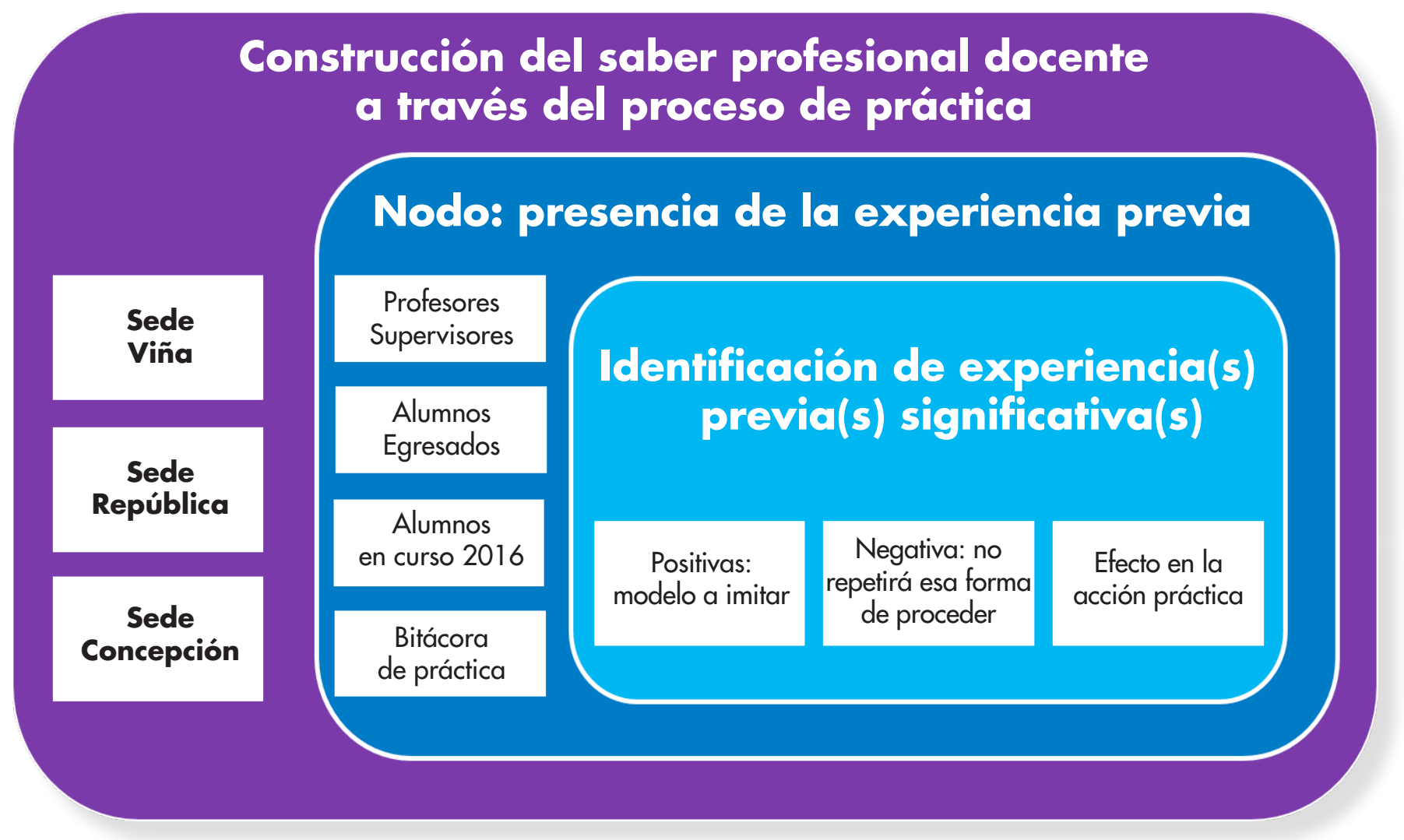

Figura 2 Análisis Categoría / Subcategoría (ejemplo)

Recogimos la apreciación desde la perspectiva de los profesores supervisores, como lo expresan algunos:

“..., muchos tuvieron maestros que los marcaron, tal vez no muchos, pero alguno que los marcó, y eso naturalmente, deja una marca, una huella. (CON-SUP-HIS-SP $)^{1}$

Normalmente los practicantes replican su experiencia como alumno, la cual se ve en una clase meramente frontal. (VIN-SUP-MAT-YL); me ha tocado ver alumnos que les cuesta deshacerse de esa mirada tradicional sobre el quehacer del profesor..., el peso de las experiencias que traen consigo es muy fuerte....ii (REP-SUP-LEN-KM)

Si abordamos este aspecto desde la mirada de los egresados encontramos también evidencias de estas experiencias previas que van dejando huella en las vivencias de los alumnos.

En este caso, las experiencias previas se remontan a la enseñanza básica y media, y abarcan la amplitud emocional que va de lo positivo a lo negativo, y lo suficientemente fuertes como para ser recordadas años después:

"Y me acordaba de la clase que más me gustaba y la trataba de replicar de la misma forma..., al menos yo así lo hacía. Por ejemplo, a mí me encantaba la historia. Tenía un profe que explicaba y como que a uno lo situaba en la etapa histórica y uno se lo recreaba todo. Yo intentaba aplicarlo de esa forma, así, enseñarles así a mis alumnas". (VINEGR-BIO-IV)

“..., yo creo que me apoyo mucho en..., en cómo me enseñaron a mi”. (VIN-EGR-BIO-IV); “..., pero él logró que me gustara, por ejemplo, los griegos, todo ese tipo de mitología, y todavía recuerdo las clases vivas; y en términos de didácticas, él es como el modelo". (CON-EGR-BIO-GG)

"Bueno, yo... secretamente pensaba "me gustaría estudiar artes para llegar y sacar esta profesora y hacer yo las clases de artes visuales"..., y hacerlas como corresponde". (CON-EGR-ART- RY)

\footnotetext{
${ }^{1}$ Las claves identifican a los participantes en la investigación. Indican la sede de origen: Concepción, República y Viña. Luego si se trata de Supervisor, Egresado o Alumno, y finalmente la especialidad e iniciales del nombre.
}

“¿Cómo llegan a ser profesores los estudiantes de pedagogía en educación secundaria en Chile? La práctica y la teoría en la formación del conocimiento profesional docente", Margot Recabarren, Cuaderno de Pedagogía Universitaria Vol. 13/ no. 26/ julio-diciembre 2016/ República Dominicana/ PUCMM/ ISSN 1814-4152 (en línea) / ISSN 1814-4144 (impresa) / p. 26-35 
EXPERIENCIAS PREVIAS:

\section{Registro-cobertura \\ Cifra indica las veces que el sujeto hace transferencia al eje de análisis}

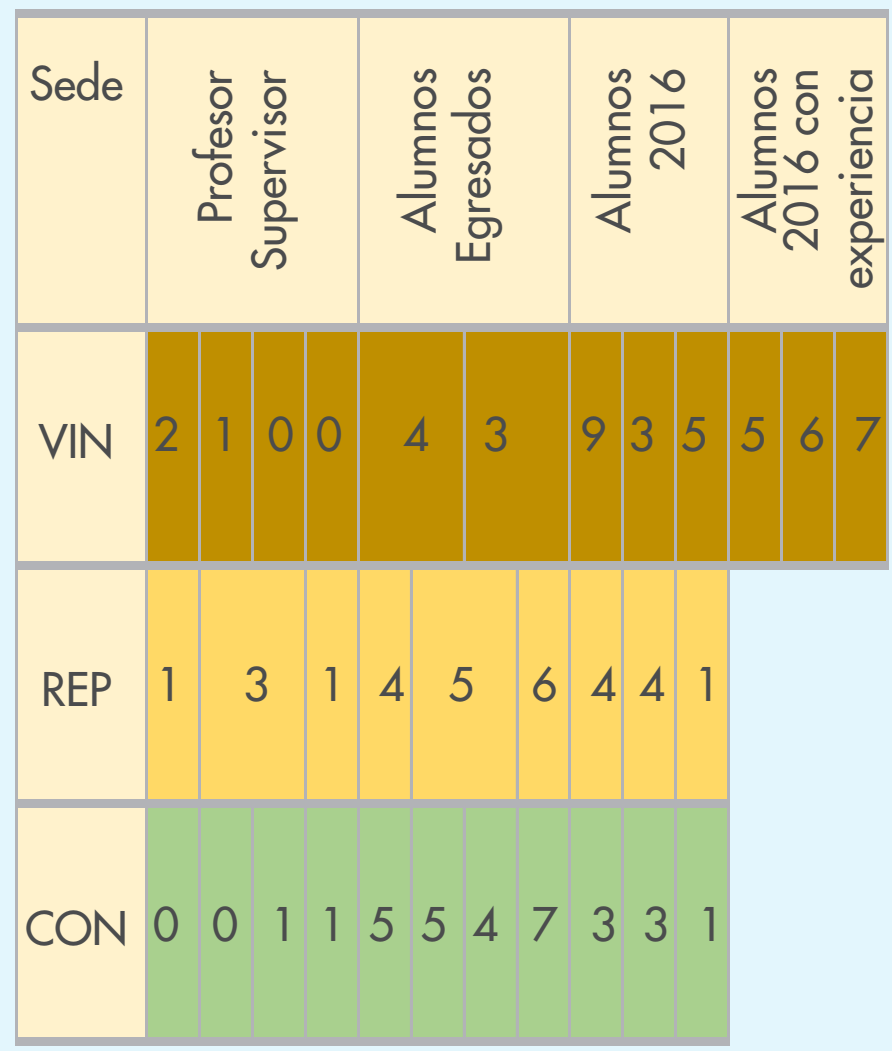

EXPERIENCIAS PREVIAS:

\section{Registro-Tipo Instrumento o registro que se utiliza ENTREVISTA-BITÁCORA-DIBUJO}

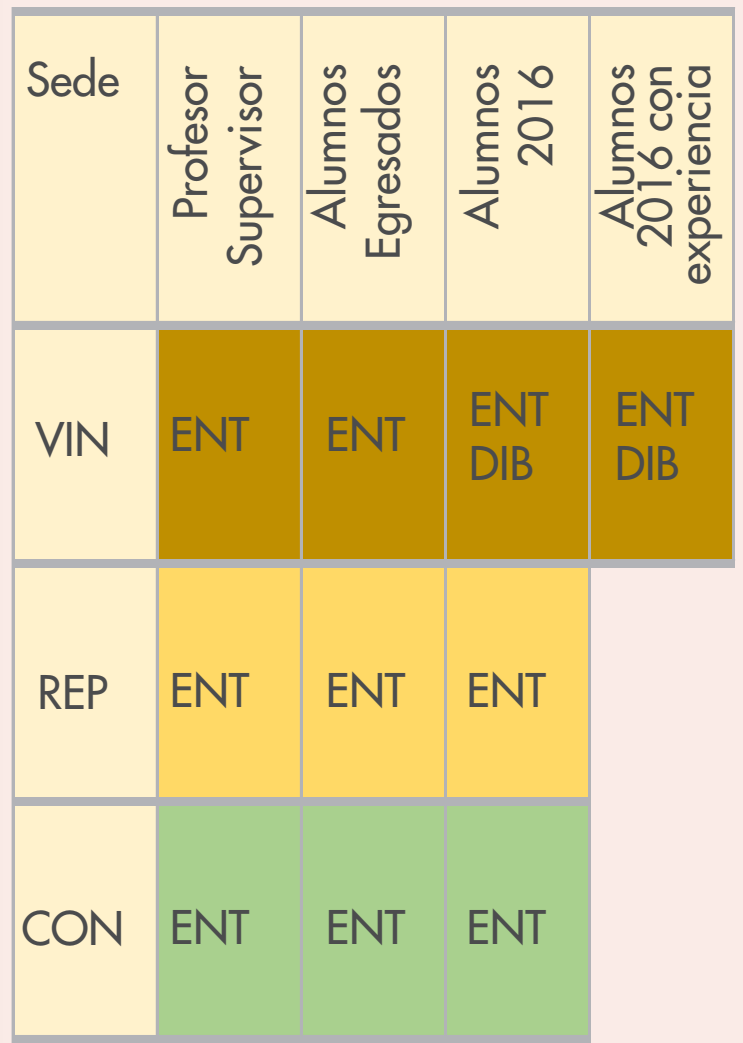

Figura 3 Registro Cobertura Categoría Experiencias Previas

Los procesos mediante los cuáles llegamos a conocer una determinada disciplina o área curricular, influirá en cómo la enseñamos.

Existen múltiples evidencias que nos muestran ciertos "arquetipos" (Marcelo, 2006) que los profesores en formación tienen respecto de la disciplina que estudian, ya sea matemáticas, lenguaje, música o educación física.

Junto al conocimiento del contenido, está el de cómo "se enseña" determinada materia o disciplina.

Desde esta perspectiva, surge el cuestionamiento: ¿Cómo hacer para contrarrestar o balancear el peso de estas experiencias previas? Alsina et al. (2016), son partidarios de una reflexiva toma de conciencia para reconocer el peso de las experiencias previas y los arquetipos. Iborra \& Nogueiras (2015), por su parte, exploran en las representaciones de maestros sobre su propia identidad profesional a partir del dibujo. Nuestra investigación también lo hizo efectivo y se llega a la conclusión que este punto de partida puede ayudar a la toma de conciencia por parte de los futuros profesores, y permitirles de-construir y re-construir su ser docente.

De acuerdo a Carlos Marcelo, el conocimiento didáctico del contenido se evidencia como el aspecto central del quehacer del profesor. A esos saberes disciplinares se agregan los pedagógicos. 
Esta integración, "representa la combinación adecuada entre el conocimiento de la materia a enseñar y el conocimiento pedagógico y didáctico referido a cómo enseñarla" (2006, p. 74). Es precisamente esa sinergia de saberes lo que transforma a un especialista en una materia, en un profesor capaz de enseñarla.

\section{La Tensión Teoría - Práctica}

Si analizamos ahora el aprendizaje de los profesores y la forma como se estructura y organiza este conocimiento, debemos partir desde algún enfoque explicativo. Debemos ubicar al sujeto profesor en el contexto que este percibe y que le da sentido, en el contexto de los procesos socioculturales que lo explican. Aprender a enseñar, o el acto de aprender, en su sentido más amplio, lo analizaremos desde el enfoque constructivo, como el "proceso que implica efectivamente la adquisición de un repertorio de conocimientos, habilidades, actitudes, creencias y afectos construidos a lo largo y ancho del ejercicio profesional de cualquier profesor" (Montero, 2001, p.153).

Repensar la formación fue una de las razones que llevó a numerosas instituciones a instaurar procesos de formación de profesores que buscan un equilibrio entre el conocimiento producido e impartido en sus aulas con respecto a la enseñanza y el aprendizaje y el conocimiento que es necesario de acuerdo a lo sugerido por los docentes en ejercicio y que es construido por ellos en su quehacer pedagógico.

Se trata entonces de entablar un proceso de diálogo entre la teoría y la práctica, de modo que el encuentro del futuro profesor con la realidad educativa, propicie una dialéctica que favorezca los aprendizajes pedagógicos. Con ese argumento, las universidades han optado por aumentar las horas de la malla curricular dedicadas a la práctica y articular estas prácticas como un eje importante en el proceso formativo, las cuáles, junto a los procesos reflexivos y de análisis instalados al interior de las prácticas, debieran ser el espacio propiciador de una adecuada construcción de aprendizajes didácticos, pedagógicos y de especialidad.

A pesar de ello, la realidad habla aún de procesos poco articulados, de esfuerzos en desarrollo, de falta de integración en algunos casos, de algunas tendencias asignaturistas, de espacios más bien destinados a generar lógicas de aplicación técnica, con pocos elementos reflexivos o analíticos, que guíen un proceso de aprendizaje desde la integración, a partir de la consideración de los contextos, de la visibilización y relevancia de la realidad.

Si estos elementos no se articulan adecuadamente, deja de reconocerse que los aprendizajes más efectivos en los futuros docentes, se legitiman cuando movilizan los saberes enseñados hacia los contextos de la práctica. Maurice Tardif lo expresa con claridad, "el trabajo no es primero un objeto que se mira, sino una actividad que se hace y, realizándola, se movilizan y construyen los saberes" (2009, p.189).

Por otra parte, resultados de investigaciones Errázuriz \& otros, 1994; Edwards \& otros, 1995, Pasmanik, 2001; Pogré, \& otros, 2003; Rival, 2005; Vaillant, 2004.), "evidencian en que en la interacción profesorestudiante, en todos los niveles del sistema educacional, predomina un estilo tradicional, frontal, vertebrado en la psiquis docente casi de manera inamovible, reacio y poco permeable al cambio". (Ibáñez, 2008, p. 9)

Esto lo evidenciaron en su experiencia directa los alumnos entrevistados, en este caso, un egresado:

"Por ejemplo, básicamente el profesor debe hacer diferentes actividades con los alumnos para que se motiven, y los profesores no hacían eso, simplemente llegaban, explicaban, el que entendía, entendía, y el que no ahí se quedaba. Entonces no había una retroalimentación, no existía esa comunicación donde el alumno le preguntaba al profesor, porque muchas veces el alumno o el estudiante temía dar una respuesta al profesor. Entonces claro, el profesor llegaba, explicaba la materia, no realizaba ejercicios o a veces nos decía "página tanto del libro, realice." Entonces no había una consecuencia entre los contenidos que él entregaba y después con las evaluaciones, también muchas veces las evaluaciones no tenían concordancia con lo que él había entregado en clases". (REP-EGR-ING-TG).

La falta de coherencia entre el discurso educativo ampliamente declarado y aceptado, y la práctica pedagógica es, a pesar de todas las evidencias, un aspecto poco investigado, y a pesar que existen investigaciones al respecto, se considera que es preciso profundizar o ampliar estos estudios (Ibáñez, 2008). Según Korthagen, "the gap between theory and practice has been a perennial issue" $(2011$, p. 32). La investigación liderada por Nolfa Ibáñez entre 2007-2008 recoge y reafirma investigaciones previas, y además concluye que una "parte importante de los indicadores de emociones desfavorables para el aprendizaje de los estudiantes, desde su propia percepción, dice relación con esta falta de coherencia". (2008, p.10)

Los alumnos entrevistados en esta investigación lo vivenciaron:

"..., claro te hablan de ser innovadores, de buscar nuevas cosas, pero tú ves que a la hora de los

“¿Cómo llegan a ser profesores los estudiantes de pedagogía en educación secundaria en Chile? La práctica y la teoría en la formación del conocimiento profesional docente", Margot Recabarren, Cuaderno de Pedagogía Universitaria Vol. 13/ no. 26/ julio-diciembre 2016/ República Dominicana/ PUCMM/ ISSN 1814-4152 (en línea) / ISSN 1814-4144 (impresa) / p. 26-35 
"quihubo"" los profesores siguen escribiendo en la pizarra y no se atreven y al final excusan "no, es que con este curso no se puede", "no, es que no hay tiempo" yo veía de repente en mi colegio que tenían los tremendos laboratorios de física, de biología, de química... los habremos usado con suerte una vez...o lo usaba el electivo, no nosotros y era como: ¿Por qué?..” (EG-VIN-ALU-ART-MG)

Los alumnos sienten a menudo que lo que ven en los colegios es la antítesis de lo que se trabaja con ellos en las asignaturas, y que las formas de proceder o las acciones que se implementan, en muchos casos no resisten un análisis desde lo pedagógico. Esto, es lo que recogen las palabras de esta alumna en práctica: ella misma se encuentra en la encrucijada de la tensión entre teoría y práctica.

"Es que de partida, el tema en cuestión siento que hay muchas cosas que..., a ver, la teoría es buena porque está bien pensada, tiene argumentos, pero se queda de alguna forma en lo ideal, no sé por qué, si bien siempre hay cosas que se pueden rescatar; pero a la hora de llevarla a la práctica siento que siempre hay como miedos de parte de los implicados en implementarla o en cosas mal hechas, no sé porque, de repente tienes toda la teoría que te dice "si, lo mejor es la educación constructivista" y llega la hora de las leyes y te pones en una cuestión súper conductista, entonces uno dice "¿Por qué? ¿Quién está ahí?"..." (VIN-ALU-ART-MG)

La relación teoría-práctica en el proceso de práctica profesional y en las vivencias de los alumnos tiene un correlato diverso y concreto. Ellos no solo perciben la dualidad teoría-práctica, sino que además la vivencian. El registro a continuación es plenamente iluminador, cuando el alumno, en un principio reconoce una acción docente como errónea y declara luego que no la realizará...

"..."mira la profe como lo hace, ¿en realidad hay que gritar o no?" "no, yo encuentro que yo no quiero ser así", "cuando yo haga clases, no voy a hacer esto (y anota), no voy a hacer esto, esto, no lo voy a hacer"..." (CON-EGR-BIO-GG)

..., pero posteriormente el mismo alumno replica esa conducta en el aula...:

Ahí entro en otra crisis, porque si bien es cierto que yo ahora estoy en ese lugar, porque estoy en el mismo lugar de mi profesora anterior... hago lo mismo que ella, de repente. Eso es una continua crítica que me hago todos los días porque todos los días levanto la voz. (CON-EGR-BIO-GG)

En este caso, bien vale preguntarse si la prevalencia de modelos tradicionales, seguramente incorporados en los años de procesos de enseñanza a lo que se vio sometida (Korthagen, 2011), prevalecieron por sobre un discurso de índole constructivista, donde la relación dialógica es relevante y donde el alumno es un agente activo, participativo y comprometido con su aprendizaje. ¿Qué pasó con el discurso teórico? Al menos prevalece la mirada crítica sobre ese desempeño, pero no basta, aparentemente, para provocar un cambio en el contexto de su aula de modo que pueda lograr un adecuado ambiente de aprendizaje.

Según Carrión (2016), es relevante que el profesor "integre el pensamiento y la práctica para apreciar y observar a ese maestro como profesional reflexivo del saber,..., que ejercita activamente la responsabilidad de incidir en los demás para la configuración de una sociedad intelectual". (p.17)

Y acá, para poder nuevamente balancear el proceso formativo, entre los elementos teóricos y la vivencia práctica, bien vale acoger la propuesta de Korthagen, que plantea el "enfoque realista en la formación del profesorado" (2010a, p.86), que privilegia trabajar sobre la base de las situaciones reales, a través de intervenciones guiadas. Sabemos que este proceso es de tal complejidad y trascendencia que la formación de cada futuro profesor debiera estar enmarcado en esta propuesta reflexiva, y abordarse desde una perspectiva integral, en una comunidad de aprendizaje y a la vez en forma personalizada, con los apoyos y mediación necesarias para potenciar al mejor nivel alcanzable, la formación de cada profesor, entregándole herramientas de autonomía, reflexivas e investigativas como para que pueda seguir creciendo autónomamente en su proceso formativo.

\section{Conclusión}

Los docentes ante el saber... ¿Cómo construyen ese saber? A pesar de que es válido asumir que el docente de educación media o secundaria desarrolla los aprendizajes disciplinares desde la especialidad, en lo que se conoce como conocimiento formal, y que muchos profesores se esmeran en trasmitir a sus alumnos a través de la frase "pasar la materia"; no queda claro cuál es el tipo de conocimiento profesional pedagógico que el futuro profesor construye durante su proceso de práctica. Esto evidencia claramente que el proceso de práctica, por su gravitación en la formación, es un proceso que es preciso re-pensar y fortalecer.

\footnotetext{
2 “quihubo”: pronunciación coloquial de la expresión “¿qué hubo?”. Hace referencia al momento en que es preciso dar cuenta de lo que se hace. También se usa como expresión de saludo, para saber de la otra persona.
}

“¿Cómo llegan a ser profesores los estudiantes de pedagogía en educación secundaria en Chile? La práctica y la teoría en la formación del conocimiento profesional docente", Margot Recabarren, Cuaderno de Pedagogía Universitaria Vol. 13/ no. 26/ julio-diciembre 2016/ República Dominicana/ PUCMM/ ISSN 1814-4152 (en línea) / ISSN 1814-4144 (impresa) / p. 26-35 
En esta perspectiva, resulta relevante averiguar entonces, cómo este alumno en su proceso de práctica, va adquiriendo las formas y procedimientos que son propias del quehacer del profesor, cómo se genera en la interacción con docentes de diferentes grados de experiencia y de experticia, en un contexto que posee dinámicas propias y distintivas que son transversales al rol docente en cualquier contexto. El alumno en práctica, en este proceso de acercamiento e inmersión de esta realidad, se siente a menudo conflictuado interiormente a medida que vivencia diferentes desempeños y se va apropiando de ellos. El practicante va incorporando una serie de acciones y pasos, secuencias que a veces sigue, en ocasiones sin mayor reflexión, actos que llegan finalmente a formar parte de su propio repertorio de rutinas adquiridas.

Fred Korthagen (2010), analizando la esencia del comportamiento profesional y del aprendizaje profesional, plantea, sobre la base de investigaciones y estudios, que a menudo los profesores aprenden desde la imprecisión, esto es, muchas de las decisiones que los docentes toman en el aula, o frente a situaciones pedagógicas, son lo que Eraut (1994, citado en Korthagen, 2010) denomina "instantáneas", esto es, se realizan de manera inconsciente o semi-consciente. El alumno en proceso de práctica observa esto y adquiere a menudo, prácticas que no siempre es capaz de respaldar con elementos teóricos de base. "Según Shavelson \& Stern (1981) y Yinger (1986), estas rutinas del profesorado se basan en gran parte en la formación de hábitos. Schön afirma: 'A menudo no somos conscientes de haber aprendido a hacer estas cosas; simplemente las hacemos'". (1983, p. 54)

Los procesos que debe monitorear el alumno en práctica, las situaciones del contexto educativo, los problemas prácticos del aula, ya sea que se refieran a situaciones individuales de aprendizaje o a situaciones de grupo, exigen un tratamiento específico, porque en buena medida son problemas singulares y particulares, aun cuando pueda haber muchos de ellos que sean similares, pero estas situaciones están fuertemente condicionadas por las características situacionales del contexto, así como por la propia historia del centro educativo, del aula específicamente como grupo y del alumno protagonista de la situación. La práctica reflexiva a partir del modelo de Korthagen ofrece una posibilidad cierta de trabajo en casos como el descrito, ya que posibilita generar un ciclo de análisis, a partir de la identificación concreta de los hechos que el alumno vivencia en los procesos de práctica.
La identificación concreta del hecho -objeto de análisis- como punto de partida, posibilita el análisis y la reflexión sobre la interrelación entre teoría y práctica. Desde este enfoque realista, según Alsina, se requiere:

1. Generar interrogantes, que emergen de la misma práctica y que el maestro en formación experimenta en un contexto real de aula.

2. Fomentar una reflexión sistemática a través del modelo ALACT: Práctica, Revisión de la Práctica, Toma de Conciencia de lo esencial, Creación de Métodos de Acción Alternativos, Ensayo.

3. Considerar que el aprendizaje es un proceso social e interactivo.

4. Fomentar la autonomía y la construcción autorregulada de las competencias profesionales. (Alsina, 2016, p. 33-34)

En resumen, tenemos entonces que el alumno, en el contexto de la singularidad de su proceso de práctica, a su vez debe convivir con una serie de actos pedagógicos y procesos en permanente desarrollo, en los cuales, la toma de decisiones o las respuestas que se producen no son siempre conscientes, a menudo espontáneas y poco relacionadas con los corpus teóricos que ha debido asimilar en su proceso formativo académico.

Las experiencias previas que trae consigo cada futuro profesor tienen una presencia poco visibilizada en esta construcción, lo que no significa que no graviten en la construcción del ser docente. A esto se suma los aprendizajes que el alumno construye en esta conflictiva relación entre la teoría y la práctica en su propio proceso de práctica inicial y profesional.

Una toma de conciencia de los elementos previos y arquetipos que trae consigo y una propuesta reflexiva e integral bajo la propuesta de Korthagen son algunas de las respuestas que pueden ayudar en este complejo proceso formativo.

\section{Referencias}

Alsina, A., Batllori, R., Falgás, M., Güel, R., Vidal, I. (2016). ¿Cómo hacer emerger las experiencias previas y creencias de los futuros maestros? Prácticas docentes desde el modelo realista. REDU, 14(2), 11-36. Recuperado de: https://dialnet. unirioja.es/ejemplar/446737 
Ávalos, Beatrice; Sotomayor, Carmen, (2011) Cómo ven su identidad los docentes chilenos, Revista Perspectiva Educacional, Vol 51, № 1. 57-86

Calvo, G. \& Camargo A., M. (2015). Hacer escuela en la formación de docentes nóveles. Páginas de Educación, 8 (1) Recuperado de: http://www.scielo. edu.uy/scielo.php?script=sci_arttext\&pid=S1688$74682015000100002 \&$ lang=pt

Carrión, W., (2016). Aspectos fundamentales que definen al maestro y su rol. Cuaderno de Pedagogía Universitaria, Año 12 / N.24 / julio-diciembre 2015 / Santiago, República Dominicana / PUCMM / p. 1420 Recuperado de: http://cuaderno.pucmm.edu.do

Castiblanco, O. Vizcaíno, D. (2010). Reseña Libro: Introducción a la Investigación Cualitativa, de Uwe Flick. ISSN 2145-4981. Vol 5 No 2 Diciembre 2010. Recuperado de: http://comunidad.udistrital.edu.co/ geaf/files/2012/09/2010Vol5No2-007.pdf

Comisión de las comunidades europeas. (2007). Comunicación de la Comisión al Consejo y al Parlamento Europeo: Mejorar la Calidad del Profesorado. Comisión de las Comunidades Europeas. Bruselas, 3.8.2007. COM (2007)392 final. Recuperado de: www.ub.edu/obipd/ PDF\%20docs/Formaci\%C3\%B3\%20Permanent/ Educaci\%C3\%B3\%20Primaria/Publicacions/ Mejorar $\% 201$ a $\% 20$ calidad $\% 20$ de $\% 201 a \% 20$ formaci\%C3\%B3n\%20del\%20profesorado.pdf

Eraut, M. (1994). Developing professional knowledge and competence. Londres: The Falmer Press.

Flick, U. (2014). La gestión de la calidad en investigación cualitativa. Madrid: Morata.

Ibáñez S., N. (2008). Saber pedagógico y práctica docente: estudio en aulas de educación parvularia y básica. (Informe final Proyecto FONIDE N²74 - 2006). Recuperado del sitio en internet de MINEDUC: $\quad$ http://sgdce.mineduc.cl/descargar. php?id_doc $=201208211124310$

Imbernon, F. (ed. 2007). La formación y el desarrollo profesional del profesorado: hacia una nueva cultura profesional. España: Graó.

Korthagen, F. (2010). La práctica, la teoría y la persona en la formación del profesorado. Revista
Interuniversitaria de Formación del Profesorado, 68 $(24,2)$, 83-101.Recuperado de: www.aufop.com/ aufop/uploaded_files/articulos/1279236671.pdf

Korthagen, F. (2011). Making teacher education relevant for practice: the pedagogy of realistic teacher education. ORBIS SCHOLAE, 5(2), 3150. Recuperado de: http://www.orbisscholae.cz/ archiv/2011/2011_2_02.pdf .

Manso A., Jesús, (2012). La Formación Inicial del Profesorado de Educación Secundaria. Análisis y valoración del modelo de la LOE (Tesis doctoral inédita, Universidad Autónoma de Madrid). Recuperado de: http://repositorio.uam.es/bitstream/ handle/10486/10818/54030_TESIS\%20 J. MANSO $\% 202012 \% 20-\% 20 U A M$.pdf?sequence=1

Marcelo G., C, (2006). La formación docente en la sociedad del conocimiento y la información: avances y temas pendientes. Ponencia presentada al IV Encuentro Internacional de KIPUS. Políticas públicas y formación docente, Isla Margarita. Recuperado de: https://idus.us.es/xmlui/bitstream/ handle/11441/17141/file_1.pdf?sequence $=1$

Montero, L. (2001). La construcción del conocimiento profesional docente. Rosario, Santa Fe: HomoSapiens.

OCDE (2014). Regards sur l'éducation 2014: Les indicateurs de l'OCDE. Recuperado de: http:// www.oecd.org/fr/edu/rse.htm

Rittershaussen, S. (2001). Chile: Innovaciones en la formación de profesores de educación media en la Pontificia Universidad Católica. Recuperado de:http://www.ibe.unesco.org/curriculum/ LatinAmericanNetworkPdf/maldorepcl2.pdf

Rittershaussen, S., Jerez, O. Contreras, I. (marzo 2015). Experiencias Internacionales de Formación Consecutiva de Profesores de Educación Secundaria. Ponencia presentada en el seminario de formación consecutiva, PUCV.

Schön, D. A. (1983). The reflective practitioner: How professionals think in action. New York: Basic Books-

Tardif, M. (2009). Los Saberes del Docente y su Desarrollo Profesional. Madrid: Narcea

“¿Cómo llegan a ser profesores los estudiantes de pedagogía en educación secundaria en Chile? La práctica y la teoría en la formación del conocimiento profesional docente", Margot Recabarren, Cuaderno de Pedagogía Universitaria Vol. 13/ no. 26/ julio-diciembre 2016/ República Dominicana/ PUCMM/ ISSN 1814-4152 (en línea) / ISSN 1814-4144 (impresa) / p. 26-35 